\title{
Transient Voltage Overshoots of High Voltage ESD Protections Based on Bipolar Transistors in Smart Power Technology
}

\author{
A. Delmas ${ }^{1,3,4}$, A. Gendron ${ }^{2}$, M. Bafleur ${ }^{3,4}$, N. Nolhier ${ }^{3,4}$ and C. Gill ${ }^{2}$ \\ ${ }^{1}$ Freescale Semiconducteur, 134 Av. du General Eisenhower, 31023 TOULOUSE Cedex, FRANCE \\ ${ }^{2}$ Freescale Semiconductor, 2100 E. Elliot Road, TEMPE AZ 85284, USA \\ 3 CNRS; LAAS; 7 Avenue du Colonel Roche, F-31077 TOULOUSE, FRANCE \\ ${ }^{4}$ Université de Toulouse; UPS, INSA, INP, ISAE, LAAS, F31077 TOULOUSE, FRANCE
}

\begin{abstract}
Transient voltage overshoots of a high voltage $(20 \mathrm{~V})$ ESD clamp based on bipolar transistors in a smart power technology are studied using different TLP pulse conditions (rise time, voltage amplitude). The physical mechanisms involved during the ESD clamp turn-on are thoroughly analyzed by the mean of TCAD simulations, allowing the definition of a set of design guidelines for the overshoot reduction.
\end{abstract}

Index Terms - Electrostatic discharges (ESD), device physics, bipolar simulation, smart power technologies.

\section{INTRODUCTION}

Efficiently protecting smart power technologies against ESD is particularly challenging for two main reasons: the need to clamp the discharge current under high voltages and the narrowing of the ESD design window [1]. To overcome the first issue, efficient protection devices are bipolar-based ones that allow the power dissipation within the semiconductor volume. Regarding the ESD design window, it is defined on the lower limit by the supply voltage of the integrated circuit and on the upper limit by the breakdown voltage (generally the gate oxide one) of the device to be protected. Typically, ESD designers rely on TLP characteristics to check if their protection is fitting in the design window. However, such characteristics are quasi-static and do not capture fast voltage transients, which can be harmful for the device to be protected [2].

A major drawback of bipolar devices in smart power technologies is their dynamic response that can induce voltage overshoot upon the ESD stress. The physical mechanisms inducing this overshoot have to be thoroughly characterized and understood both to take it into account the ESD design window and to improve the dynamic performance of the protection device.

In the following, section II presents the technology and the protection device under study. Section III provides measurement results of the overshoot behavior under various types of TLP pulses. Section IV analyzes the device behavior during the overshoot and proposes design guidelines to minimize it.

\section{ESD PROTECTION DEVICE}

This study is carried out for an ESD clamp targeting the protection of $20 \mathrm{Volts} \mathrm{I} / \mathrm{Os}$ in a $0.25 \mu \mathrm{m}$ smart power technology featuring a low-doped $\mathrm{P}^{-}$-epitaxy, a high-doped $\mathrm{P}^{+}$-substrate, a high-doped $\mathrm{N}^{+}$-buried layer and deep trench isolation.

\section{A. Device description}

The device is an implementation of bipolar transistors providing a protection against positive and negative ESD (Fig. 1). It is formed by two P-wells isolated from each other by a deep $\mathrm{N}$-well connected to an $\mathrm{N}^{+}$-buried layer (NBL). Shallow highly-doped $\mathrm{N}$ and P-type diffusions are located in each $\mathrm{P}$-well. The whole device is surrounded by a $\mathrm{N}$-well isolation ring.

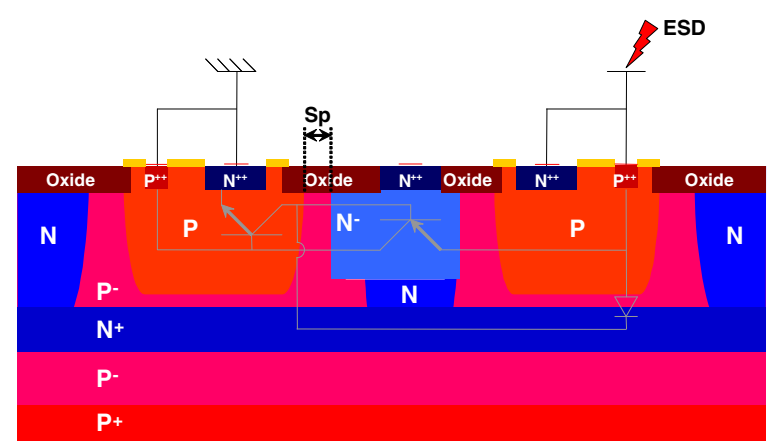

Fig. 1. Simplified cross-section of the 20 V ESD clamp under test.

In this study, we will only consider a positive ESD event. Given this condition, three bipolar transistors have to be taken into account: a lateral NPN having the P-well tied to the ground as the base, the shallow $\mathrm{N}$ diffusion in this $\mathrm{P}$-well as the emitter and the middle N-well as the collector, a vertical NPN having the same emitter and base as the lateral NPN but the NBL as the collector and a lateral PNP having the base and collector of the lateral NPN as respectively its collector and base and the P-well tied to the signal as the emitter.

The triggering of the protection relies on the selfbiasing effect in the lateral NPN transistor. Basically, 
when the voltage reaches the avalanche breakdown of the reverse biased base/collector junction (N-well/Pwell junction), holes are injected into the base and progressively build up a potential drop across the emitter/base junction. Eventually, the transistor turnson, and the voltage drops as the electrons injected by the emitter contributes to the avalanche. Concurrently, the vertical NPN gets also triggered as it shares its emitter-base junction with the lateral NPN. The triggering voltage $\left(\mathrm{V}_{\mathrm{t} 1}\right)$, which is closely related to the avalanche breakdown voltage, can be properly adjusted through the spacing ( $\mathrm{Sp}$ ) between $\mathrm{N}$-well and P-well masks.

The lateral NPN turning-on tends to induce a SCR effect by biasing the lateral PNP. However, high current densities are required to get the full SCR effect. At low current, the bipolar gain of the PNP is too low (lower doping concentration for P-well than $\mathrm{N}$-well) to provide an effective feedback. Such behavior allows leveraging the SCR to get a low onstate resistance $\left(\mathrm{R}_{\mathrm{ON}}\right)$ and a high robustness, while keeping a high holding voltage $\left(\mathrm{V}_{\mathrm{H}}\right)$.

\section{B. TLP Characterization}

Quasi-static transmission line pulse (TLP) measurements (100 ns pulse width, 10 ns rise time) were performed on 75 m-long devices (Fig. 2). The results exhibit a $\mathrm{V}_{\mathrm{t} 1}$ of $28.9 \mathrm{~V}$ and $\mathrm{a} \mathrm{V}_{\mathrm{H}}$ of $12.5 \mathrm{~V}$. The device gets damaged for a current $\left(\mathrm{I}_{\mathrm{t} 2}\right)$ of $6.9 \mathrm{~A}$, corresponding to an increase of the leakage current.

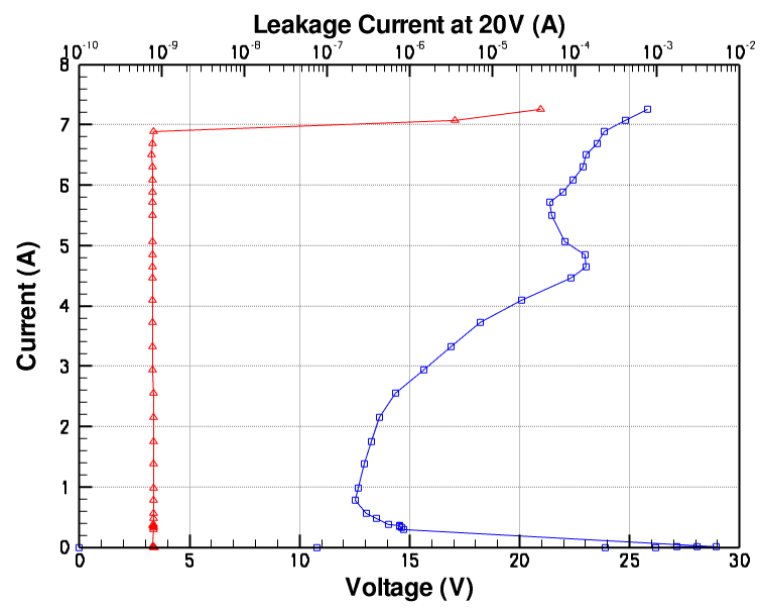

Fig. 2. TLP characteristic of $20 \mathrm{~V}$ ESD clamp.

\section{TRANSIENT VOLTAGE OVERSHOOT CHARACTERIZATION}

\section{A. Description of the measurement set-up}

A dedicated experimental setup has been developed by the authors [3] to provide a precise characterization of the transient behavior of ESD protections. This "transient TLP" (t-TLP) tool is based on a modified $50 \Omega$ very fast Transmission Line Pulsing (vf-TLP) measurement setup, for which the frequency response has been characterized and used as a correction function for scope raw data. It allows the extraction of $\mathrm{V}(\mathrm{t})$ and $\mathrm{I}(\mathrm{t})$ characteristics for pulse voltages up to $1000 \mathrm{~V}$ and pulse widths from 1.25 to $100 \mathrm{~ns}$. The time resolution is $60 \mathrm{ps}$ and the voltage error less than $4 \mathrm{~V}$. Even though the purpose of the tTLP tool development was to characterize the overshoot of the ESD protections, it can also be used as a precise "quasi-static" TLP/vf-TLP tester, by averaging $\mathrm{V}(\mathrm{t})$ and $\mathrm{I}(\mathrm{t})$ waveforms in the last nanoseconds. Then a set of I-V points is obtained for every TLP pulse voltage, as in the conventional TLP setup.

\section{B. Experimental Results}

$\mathrm{t}$-TLP measurements were performed on the $20 \mathrm{~V}$ clamp for 1 and $10 \mathrm{~ns}$ rise times $\left(\mathrm{t}_{\mathrm{r}}\right)$. For each pulse, the maximum voltage $\left(\mathrm{V}_{\text {Max }}\right)$ reached by the protection during the triggering was extracted (Fig. 3). $\mathrm{V}_{\text {Max }}$ values grow continuously with the current, up to approximately $37 \mathrm{~V}$ at $6 \mathrm{~A}$ for $10 \mathrm{~ns}$ rise time and 50 $\mathrm{V}$ for $1 \mathrm{~ns}$ rise time. Compared to $\mathrm{V}_{\mathrm{t} 1}$, it corresponds to, respectively, $8 \mathrm{~V}$ and $21 \mathrm{~V}$ overvoltages, which have to be taken into account for the design window.

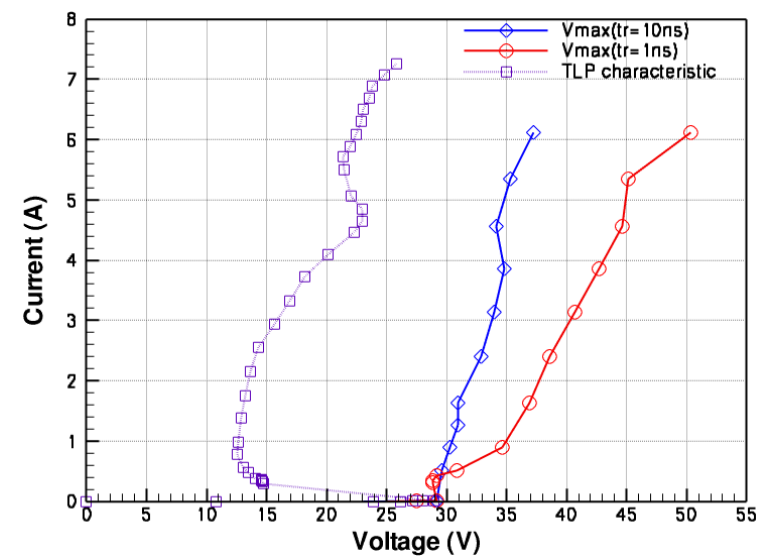

Fig. 3. I- $\mathrm{V}_{\mathrm{Max}}$ curves with I the quasi-static TLP current for $100 \mathrm{~ns}$ pulse width and $\mathrm{V}_{\mathrm{Max}}$ the peak voltages for both 10 $\mathrm{ns}$ and $1 \mathrm{~ns}$ rise times. The quasi-static TLP characteristic is plotted as a reference.

\section{ANALYSIS OF THE DEVICE OPERATION DURING THE OVERSHOOT}

\section{A. Time dependent conditions for the voltage snapback}

To determine the condition for a device to snapback during a TLP pulse, we will consider the equivalent circuit of the tester presented in Fig. 4. The condition for the voltage to reach its maximum can be written as:

$$
\frac{d V_{D U T}}{d t}=0
$$

where $\mathrm{V}_{\text {DUT }}$, the voltage across the device, is equal to:

$$
V_{\text {DUT }}=V_{\text {gen }} \quad R_{\text {ser }} I_{\text {DUT }}
$$


with, $V_{\text {gen }}$ the TLP source voltage, $R_{\text {ser }}$ the series resistor in the equivalent circuit of the TLP, typically 50 , and $\mathrm{I}_{\mathrm{DUT}}$ the current through the device. By combining equations (1) and (2), we obtain the snapback condition:

$$
\frac{d\left(R I_{D U T}\right)}{d t}=\frac{d V_{g e n}}{d t}
$$

This condition means that the increase of the TLP source voltage is compensated by the increase of the voltage drop induced by the DUT current through the TLP resistance. Basically, the measurement set-up impacts the overshoot through both the voltage slope and the series resistance. We can notice that, when the TLP pulse reaches its plateau, the ESD device can easily snap back as $\mathrm{dV}_{\text {gen }} / \mathrm{dt}$ is equals to zero. However, in this particular case, the overshoot is directly correlated to the shape of the TLP pulse and do not represent the device behavior during a real event (HBM for example).
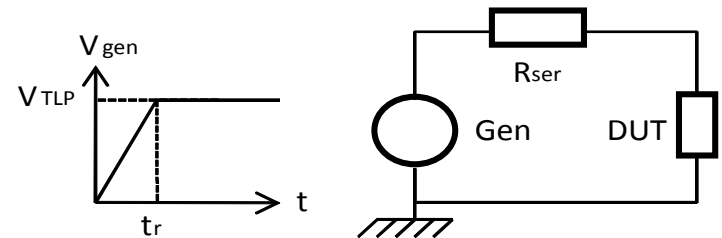

Fig. 4. Simplified version of a TLP tester equivalent circuit

\section{B. TCAD simulations results}

In order to investigate the physical behavior of the ESD device during the overshoot, two dimensional device simulations were performed using Synopsys TCAD tools. First, the device doping cross-section was generated by process simulation. Then, a particular care was taken in selecting the physical models required for accurate electro-thermal simulations of ESD devices. As part of this calibration process, the electrons and holes lifetimes were adjusted to fit the bipolar gain at low current, as recommended in [4].

The simulation results for $100 \mathrm{~V}$ TLP pulses with 1 ns and 10 ns rise times are presented in Fig. 5. Up to $\mathrm{V}_{\text {Max }}$, the agreement with measurements is very good (the precision on $\mathrm{V}_{\text {Max }}$ value is $4 \%$ and $1.5 \%$ for $1 \mathrm{~ns}$ and $10 \mathrm{~ns}$ rise times, respectively). After snapback, the voltage does not drop as fast in simulation as in measurement. This discrepancy can be attributed to the inaccuracy of the generation model under high current conditions. However, as long as we focus on the physical mechanisms controlling the peak voltage $\mathrm{V}_{\mathrm{Max}}$, the simulation study can be used with confidence to get a deeper insight into the involved phenomena.

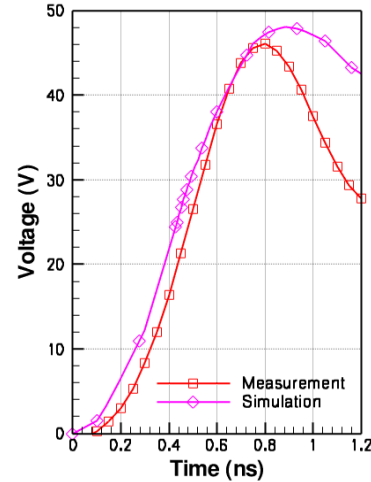

(a)

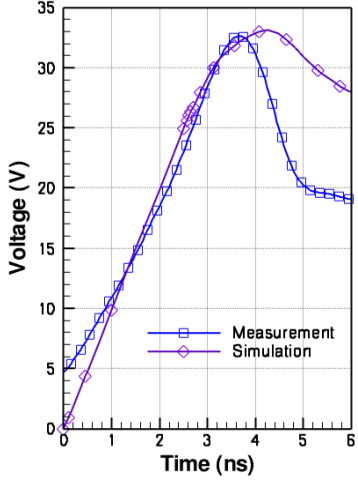

(b)
Fig. 5. Measured and simulated voltage waveforms at the beginning of a $100 \mathrm{~V}$ TLP pulse for $1 \mathrm{~ns}$ (a) and $10 \mathrm{~ns}$ (b) rise times.

We can notice the peak voltages are reached before the end of the TLP voltage rise, for $\mathrm{t}<\mathrm{t}_{\mathrm{r}}$. In these cases, the overshoot is not impacted by the change in the slope of the TLP pulse when it reaches its plateau.

\section{Physical mechanisms controlling the overshoot}

In the following of the study, we will focus on the simulated voltage waveform for $10 \mathrm{~ns}$ rise time (Fig. 5 b).

The first parameter of interest is the delay for the ESD device to go into snapback after it reaches its DC breakdown voltage $(\mathrm{BV})$, at the time $\mathrm{t}_{\mathrm{Bv}}$. A static simulation gave a value of $24.9 \mathrm{~V}$ for $\mathrm{BV}$. By reporting it on the voltage waveform, we get a delay of $1.77 \mathrm{~ns}$ during which the voltage increases by $8.2 \mathrm{~V}$ (Fig. 6).

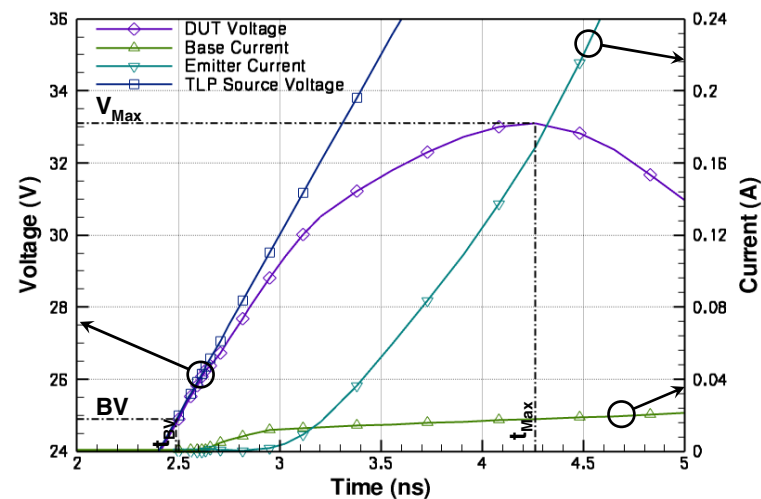

Fig. 6. Simulated voltage and current waveforms next to the peak voltage.

To start getting insight into the behavior of the ESD device during this delay, we have plotted the emitter and base current waveforms (Fig. 6). The base current starts increasing immediately after time $t_{\mathrm{BV}}$, thanks to avalanche holes generation, followed after approximately $400 \mathrm{ps}$ by the emitter current, but very slowly at the beginning (it reaches $10 \mathrm{~mA}$ with a delay of 630 ps during which the voltage has already increased by $5.2 \mathrm{~V}$ ). The emitter current gradually builds up, reaching higher values and counteracting more efficiently the TLP voltage increase. When the 
device finally snaps back, the emitter current is already relatively high, close to $170 \mathrm{~mA}$.

The delay for the emitter current to set up corresponds to the time required for the avalanche current to bias the emitter-base junction, or, in other words, to charge the emitter-base junction capacitance, as was described in [5]. Based on these observations, a first set of design guidelines can be defined to reduce the overshoot: increasing the P-well resistance (for example by pushing the base contact away from the emitter or adding an external resistance) and reducing the emitter-base junction capacitance (for example by designing a smaller emitter).

When the emitter-base junction becomes forward biased, the lateral NPN transistor can enter in its selfbiased mode. The remaining delay for the device to snap back will depend on how strong the feedback is between the electron current coming from the emitter and the avalanche at the base-collector junction. Under these conditions, the emitter injection ratio (Fig. 7 a) and the electrons base transit time (Fig. 7 b) are critical as they set, respectively, the efficiency of the emitter injection and the time constant of the feedback. The low value of the injection ratio at 3.20 ns means that the emitter-base diode has not been triggered yet and the decreasing trend observed after reaching a maximum value is typical of high current effects. The variations in the values of the base transit time are also related to high current effects.

A second set of design guidelines for the overshoot reduction can be defined targeting an increase of the emitter injection ratio and a decrease of the electrons base transit time. However, such rules have to be applied with care as they will tend to reduce the holding voltage [6].

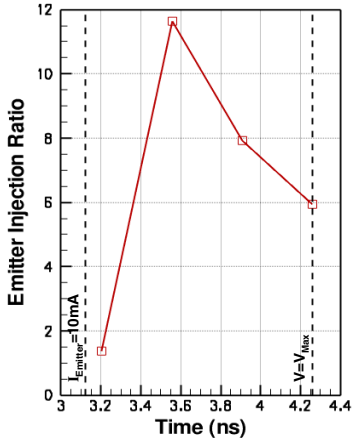

(a)

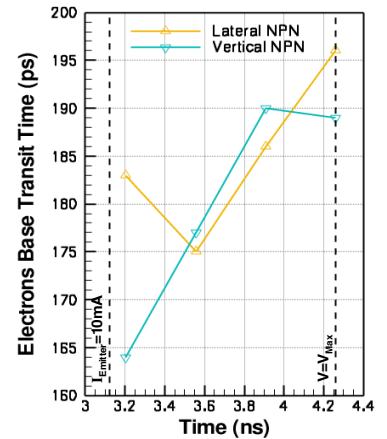

(b)
Fig. 7. Emitter Injection (a) and electrons base transit times for the lateral and vertical NPN (b).

In addition, Fig. $7 \mathrm{~b}$ shows that the base transit times for the lateral and vertical NPN are close to each other. This leads to a split of the base current between the lateral and the vertical paths (Fig. 8). As a result, some of the electrons injected by the emitter do not flow through the high avalanche region located at the lateral base-collector junction. The vertical NPN, which has a very positive impact on the robustness, turns out to slow down the snapback.

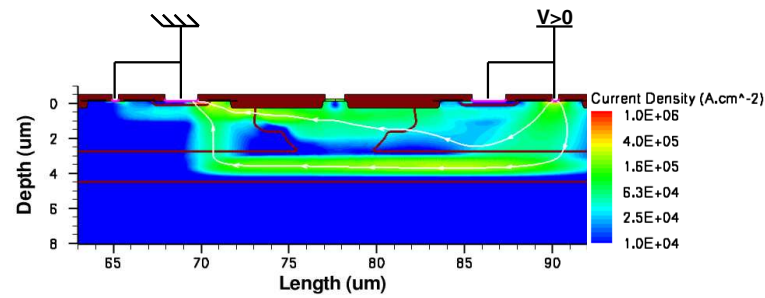

Fig. 8. Current density distribution at $\mathrm{t}\left(\mathrm{V}=\mathrm{V}_{\mathrm{Max}}\right)$ showing the lateral and vertical current paths.

\section{CONCLUSION}

The transient turn-on of a 20 Volts ESD clamp based on bipolar transistors has been characterized by the mean of a home-made transient ESD tester. Voltage overshoots above $\mathrm{V}_{\mathrm{t} 1}$ have been reported, up to approximately $30 \%$ for $10 \mathrm{~ns}$ rise time and $70 \%$ for $1 \mathrm{~ns}$ rise time. By leveraging TCAD simulations, the overshoot was divided into two segments. The first one corresponds to the time required to forward bias the emitter-base junction, and the second to the gradual increase of the emitter current in a self-biased NPN transistor. Thanks to these results, design guidelines for the overshoot can be defined by targeting: the increase of the P-well resistance, the decrease of the emitter-base junction capacitance, the increase of the emitter injection ratio and the decrease of the electrons base transit time.

\section{REFERENCES}

[1] M.P.J. Mergens, M.T. Mayerhofer, J.A. Willemen, M. Stecher, "ESD Protection Considerations in Advanced High-Voltage Technologies for Automotive", in Proc. EOS/ESD Symposium, pp. 54-63, September 2006.T.

[2] T. Smedes, and N. Guitard, "Harmful voltage overshoots due to turn-on behaviour of ESD protections during fast transient", in Proc. EOS/ESD Symposium, pp. 357-365, September 2007.

[3] A. Delmas, D. Tremouilles, N. Nolhier, M. Bafleur et al., "Accurate Transient Behavior Measurement of High-Voltage ESD Protections Based on a Very Fast Transmission-Line Pulse System," in Proc. EOS/ESD Symposium, September 2009.

[4] K. Esmark, "Device Simulation of ESD Protection Elements", Series in Microelectronics, vol.128, ISBN 3-89649-781-2, 2002.

[5] J. Di Sarro, K. Chatty, R. Gauthier and E. Rosenbaum, "Study of Design Factors Affecting Turn-on Time of Silicon Controlled Rectifiers (SCRs) in 90 and $65 \mathrm{~nm}$ Bulk CMOS Technologies," in Proc. IRPS 2006, pp. 163-168.

[6] A. Gendron, P. Renaud, P. Besse, C. Salamero et al., "Area-Efficient Reduced and No-Snapback PNP-based ESD Protection in Advanced Smart Power Technology," in Proc. EOS/ESD Symposium, pp. 6976, September 2006. 